\title{
A NOTE ON MORE INEQUALITIES FOR SECTOR MATRICES
}

\author{
JUNJIAN YANG, LINZHANG LU AND ZHEN CHEN
}

Abstract. In this note, we correct an inequality and a proof of another result due to Liu and Wang

[Bull. Iranian Math. Soc. 2018; 44: 1059-1066].

Mathematics subject classification (2010): 47A63, 15A45.

Keywords and phrases: Sector matrices, unitarily invariant norm, arithmetic mean, geometric mean, harmonic mean.

\section{REFERENCES}

[1] R. Bhatia, Matrix analysis, GTM 169, Springer, New York, 1997.

[2] R. Bhatia, Positive Definite Matrices, Princeton University Press, Princeton, 2007.

[3] S. W. DRURY, Principal powers of matrices with positive definite real part, Linear Multilinear Algebra. 63 (2015), 296-301.

[4] S. DRURY, M. LIN, Singular value inequalities for matrices with numerical ranges in a sector, Oper. Matrices, 8 (2014), 1143-1148.

[5] X. FU, Y. LIU, Rotfel'd inequality for partitioned matrices with numerical ranges in a sector, Linear Multilinear Algebra. 64 (2016), 105-109.

[6] C. K. LI, N. SZE, Determinantal and eigenvalue inequalities for matrices with numerical ranges in a sector, J. Math. Anal. Appl. 410 (2014), 487-491.

[7] M. Lin, Extension of a result of Haynsworth and Hartfiel, Arch. Math. 104 (2015), 93-100.

[8] M. Lin, Some inequalities for sector matrices, Oper Matrices. 10 (2016), 915-921.

[9] M. LiN, F. SUN, A property of geometric mean of accretive operators, Linear Multilinear Algebra. 65 (2017), 433-437.

[10] J. LiU, Generalizations of the Brunn-Minkowski inequality, Linear Algebra Appl. 508 (2016), 206213.

[11] J. LiU, Q. WANG, More inequalities for sector matrices, Bull. Iranian Math. Soc. 44 (2018), 1059 1066.

[12] X. Zhan, Matrix Theory, American Mathematical Society, Providence, 2013.

[13] F. ZHANG, A matrix decomposition and its applications, Linear Multilinear Algebra. 63 (2015), 20332042.

[14] Y. ZHANG, Eigenvalue majorization inequalities for positive semidefinite block matrices and their blocks, Linear Algebra Appl. 446 (2014), 216-223. 